\title{
QUALIDADE DE PRODUTOS CÁRNEOS FABRICADOS SOB INSPEÇÃO FEDERAL NO ESTADO DO PARANÁ
}

\author{
Heitor Daguer ${ }^{1}$, Heidi Derksen Silva ${ }^{2}$, Emilia Tirie Higashiyama², Cristina Maria Zanette ${ }^{3}$, Luciano \\ DOS SANTOS BERSOT ${ }^{3}$ \\ 1 - Ministério da Agricultura, Pecuária e Abastecimento. Laboratório Nacional Agropecuário. Serviço Laboratorial \\ Avançado em Santa Catarina - São José, SC \\ 2 - Ministério da Agricultura, Pecuária e Abastecimento. Laboratório Nacional Agropecuário. Serviço Laboratorial \\ Avançado - Curitiba, PR \\ 3 - Programa de Pós-Graduação em Tecnologia de Alimentos da Universidade Federal do Paraná - Curitiba, PR - \\ lucianobersot@ufpr.br
}

\section{RESUMO}

Objetivou-se com o presente estudo avaliar amostras de produtos cárneos fabricados sob inspeção federal no Estado do Paraná quanto às suas características microbiológicas, segundo a legislação vigente. De acordo com o tipo de produto, foram ainda quantificados os teores de nitrito e de amido. Todas as amostras foram coletadas nos estabelecimentos produtores e as análises foram realizadas conforme as recomendações do Ministério da Agricultura, Pecuária e Abastecimento. Das 131 amostras de produtos cárneos analisadas, cinco $(3,82 \%)$ apresentaram-se contaminadas por Salmonella sp. e três $(2,3 \%)$ encontravam-se contaminadas por coliformes fecais. Para as amostras de salsicha, os valores de amido variaram de $1,71 \%$ a $10,28 \%$. Nas amostras de mortadela, esses valores variaram de 3,01 \% a $16,5 \%$. Dez amostras de salsicha apresentaram teores de amido fora dos padrões regulamentares, assim como oito amostras de mortadela se apresentaram em não conformidade com os dispositivos legais. Os valores de nitrito residual detectados nos produtos variaram de 3,7 $\mathrm{mg} / \mathrm{kg}$ a $1.398,5 \mathrm{mg} / \mathrm{kg}$. Verificou-se que 23 amostras $(18,4 \%)$ excederam o limite regulamentar, apresentando um valor médio de $395,91 \mathrm{mg} / \mathrm{kg}$ de nitrito residual. Pelos resultados obtidos, conclui-se que apesar da boa qualidade encontrada na maioria dos produtos, alguns fabricantes demandam uma intensificação da fiscalização, de modo a atender os requisitos regulamentares de qualidade para produtos cárneos.

PALAVRAS-CHAVE: amido; carnes; nitrito; produtos cárneos.

\section{QUALITY EVALUATION OF MEAT PRODUCTS PRODUCED UNDER FEDERAL INSPECTION IN THE STATE OF PARANÁ}

The aim of this paper was to evaluate microbiological characteristics of meat products produced under federal inspection by industries in the State of Paraná (Brazil), following Brazilian regulations. According to the type of meat product, quantification of nitrite and starch was also determined. All samples were collected at the plant and all analysis were carried out according to the Brazilian Ministry of Agriculture, Livestock and Food Supply's 
recommendations. From 131 samples analyzed, five $(3.82 \%)$ were contaminated by Salmonella sp. and three $(2.3 \%)$ presented fecal coliforms. The other samples were legally acceptable. Frankfurters showed starch levels ranging from $1.71 \%$ to $10.28 \%$. In bologna, this variation ranged from $3.01 \%$ to $16.5 \%$. Residual nitrite levels in all products ranged from $3.7 \mathrm{mg} / \mathrm{kg}$ to $293.10 \mathrm{mg} / \mathrm{kg}$. An amount of 23 samples (18.4\%) had residual nitrite levels higher than legal limits, with a medium level of 395.91 $\mathrm{mg} / \mathrm{kg}$. The results found suggest that, although the analyzed samples present good sanitary quality, some producers need to be more carefully inspected, in order to attend Brazilian regulations for meat products.

KEYWORDS: inspection; meat; meat products; nitrite; quality; starch.

\section{INTRODUÇÃO}

A inocuidade e a qualidade nutricional dos produtos cárneos estão entre as principais preocupações do serviço de fiscalização de alimentos, porque tais produtos são de consumo muito popular no Brasil, entre todas as faixas etárias e níveis sociais. A produção nacional anual de embutidos ultrapassa 1,2 milhões de toneladas, destacando-se a fabricação de linguiças frescais, por sua aceitação e comercialização (MELO FILHO et al., 2004; OLIVEIRA et al., 2005).

O nitrito é um dos principais aditivos utilizados na indústria de carnes, atuando como conservante e incrementando as características sensoriais dos produtos. No entanto, o excesso de nitrito residual no produto final pode proporcionar o desenvolvimento de intoxicações, assim como a formação de nitrosaminas, compostos cancerígenos gerados a partir da reação do nitrito em excesso com aminas da carne (DAGUER, 2005). Salsichas, mortadelas e linguiças figuram entre os produtos cárneos que apresentam teor de nitritos residual superior ao estabelecido pela legislação vigente (CUNHA et al., 2003).

Em produtos de salsicharia, os amidos e féculas são profusamente empregados como ligadores. No entanto, esses ingredientes devem ser classificados como substâncias de enchimento, porque sua utilização barateia a fabricação e auxilia na retenção de água do produto, diminuindo a quantidade de matéria-prima cárnea empregada na formulação (PARDI et al., 1995).

Neste contexto, o presente trabalho teve por objetivo avaliar aspectos higiênicos, sanitários e tecnológicos de amostras de produtos cárneos fabricados sob inspeção federal no Estado do Paraná, de forma a determinar a sua qualidade microbiológica e de algumas características físico- químicas, tendo como base a legislação pertinente.

\section{MATERIAL E MÉTODOS}

Foram coletadas 131 amostras de produtos cárneos diversos em 25 estabelecimentos sob inspeção federal no Estado do Paraná, de acordo com o programa estabelecido pelo Serviço de Inspeção de Produtos Agropecuários (SIPAG), entre os meses de março e dezembro de 2006, perfazendo $52,5 \%$ da quantidade total (40) de estabelecimentos que possuem dependências para fabricação de produtos cárneos. As amostras foram transportadas sob refrigeração até o Serviço Laboratorial Avançado do Laboratório Nacional Agropecuário (LANAGRO) do MAPA, sediada na cidade de Curitiba, para realização dos exames físicoquímicos e microbiológicos. Todas as análises foram realizadas em duplicata, em até dez dias após a coleta.

As amostras de linguiças analisadas foram classificadas como frescais à base de carne suína (variedades de pernil, toscana e de carne suína) e frescais de carne de frango, totalizando 51 amostras. Dentre as linguiças cozidas e defumadas, foram analisadas 11 amostras de linguiça calabresa. As demais amostras se constituíram de produtos cozidos sendo analisadas 32 amostras de mortadela, 23 amostras de salsicha, oito amostras de apresuntado, três amostras de apresuntado tipo lanche e três amostras de presunto.

Para as análises microbiológicas foram pesados $25 \mathrm{~g}$ do produto e diluídos em $225 \mathrm{~mL}$ de solução salina peptonada $(0,1 \%)$ e homogeneizada por 2 minutos. Foram realizadas as análises de contagem de Clostridium sulfito-redutor, contagem de Staphylococcus coagulase positiva, número mais provável de coliformes fecais e pesquisa de Salmonella sp., conforme método preconizado pela Instrução Normativa $n^{\circ} 62$ do MAPA (BRASIL, 
2003). Os padrões microbiológicos utilizados para determinação da qualidade dos produtos cárneos foram baseados na RDC $n^{\circ} 12$ de janeiro de 2001 da Agência Nacional de Vigilância Sanitária (BRASIL, 2001).

As análises físico-químicas realizadas foram a quantificação de amido em embutidos cozidos (salsichas e mortadelas) e a quantificação de nitrito residual (em todos os produtos), de acordo com a Instrução Normativa 20 (BRASIL, 1999).

\section{RESULTADOS E DISCUSSÃO}

Das 131 amostras submetidas às análises microbiológicas, cinco amostras de linguiças frescais, $(3,82 \%)$ apresentaram-se contaminadas por Salmonella sp. A presença de coliformes fecais acima do previsto na legislação $(103 \mathrm{NMP} / \mathrm{mL})$ foi detectada em três amostras de salsicha (1,53\%) (Tabela 1). Produtos cárneos crus, como as linguiças frescais, frequentemente podem veicular microorganismos patogênicos em virtude da contaminação da matéria prima, condimentos e/ou manipulação inadequada e, por não serem cozidos, não permitem a destruição de células vegetativas, como Salmonella sp.

A taxa de isolamento de Salmonella sp. em linguiças frescais tem se mostrado bastante variável em diferentes estudos conduzidos no Brasil. Em contraste à presente pesquisa, SALVATORI et al. (2003) realizaram exames microbiológicos de 93 amostras de embutidos (frescais e maturados) coletadas no mercado municipal de Porto Alegre (RS), que não revelaram contaminação por Salmonella sp. No entanto, os autores evidenciaram cinco amostras com coliformes fecais acima do permitido, tendo constatado que todas aquelas amostras haviam sido coletadas no verão e encontravam-se expostas sem refrigeração. Da mesma forma, MARQUES et al. (2006), pesquisando linguiças do tipo frescal comercializadas no município de Lavras (MG), não isolaram Salmonella sp. em nenhuma das 20 amostras analisadas. Já SILVA et al. (2002), analisando 32 amostras de linguiça mista tipo frescal, comercializadas na cidade de Pelotas (RS), verificaram que $17,86 \%$ das amostras estavam contaminadas por Salmonella sp.

Tabela 1 - Resultados das análises microbiológicas das 131 amostras de produtos cárneos fabricados sob inspeção federal no Estado do Paraná, de março a setembro de 2006

\begin{tabular}{lccccc}
\hline \multicolumn{1}{c}{ Produto* } & $\mathrm{N}^{* *}$ & $\begin{array}{c}\mathrm{CSR} \\
\text { (UFC/g) }\end{array}$ & $\begin{array}{c}\mathrm{SCP} \\
(\mathrm{UFC} / \mathrm{g})\end{array}$ & $\begin{array}{c}\mathrm{CF} \\
(\mathrm{NMP} / \mathrm{g})\end{array}$ & $\begin{array}{c}\text { Salmonella sp. } \\
\text { (Pres. ou Aus/25g) }\end{array}$ \\
\hline Apresuntado (7) & 8 & 0 & 0 & 0 & 0 \\
Lanche (2) & 3 & 0 & 0 & 0 & 0 \\
Linguiça frescal (18) & 51 & 0 & 0 & 0 & 5 \\
Linguiça tipo calabresa (10) & 11 & 0 & 0 & 0 & 0 \\
Mortadela (17) & 32 & 0 & 0 & 0 & 0 \\
Presunto cozido (3) & 3 & 0 & 0 & 0 & 0 \\
Salsicha (13) & 23 & 0 & 0 & 3 & 0 \\
\hline Total & 131 & 0 & 0 & 3 & 5 \\
\hline
\end{tabular}

* Entre parênteses a quantidade de marcas analisadas por produto.

** $\mathrm{N}=$ quantidade de amostras analisadas; $\mathrm{CSR}=$ Clostrídios sulfito redutores; $\mathrm{SCP}=$ Staphylococcus coagulase positiva; $\mathrm{CF}=$ coliformes fecais

Coliformes fecais têm sido usados para determinar condições higiênicas de alimentos e presença potencial de patógenos (JAY, 2000). Na presente pesquisa, a contaminação das salsichas por esses micro-organismos caracteriza uma deficiência pós-processo, por serem as salsichas produtos cárneos submetidos ao cozimento, denotando práticas defici- entes de higiene pelos manipuladores nos estabelecimentos fabricantes dessas amostras. Coincidentemente, as três amostras contaminadas são oriundas de estabelecimentos que não dispõem de processo automatizado para descasque das salsichas após cozimento, sendo tal operação realizada manualmente, o que pode ter se constituído no principal ponto de 
contaminação dessas amostras.

Na Tabela 2 estão expostos os valores de nitrito residual encontrados nas amostras de produtos cárneos. Observou-se variação de 3,7 a 1.398,5 $\mathrm{mg} / \mathrm{kg}$ nas amostras examinadas. Em 23 amostras $(18,4 \%)$, os valores de nitrito residual ultrapassaram o limite máximo de $150 \mathrm{mg} / \mathrm{kg}$ preconizado pelo MAPA (BRASIL, 2007), caracterizando risco à saúde pública. A média de nitrito residual encontrado nas amostras que estavam em desacordo foi de $395,91 \mathrm{mg} / \mathrm{kg}$, devendo ser ressaltado que os produ- tos foram coletados nos estabelecimentos produtores com, no máximo, dois dias de fabricação. Esses altos valores encontrados sugerem uma possível utilização abusiva de nitratos e/ou nitritos. Por outro lado, baixas concentrações de nitrito em produtos cárneos também podem representar risco por não fornecerem as características de conservação esperadas, com uma possível diminuição do tempo de prateleira desses produtos e multiplicação de micro-organismos patogênicos, como Clostridium botulinum (TURRA \& AYUB, 1999).

Tabela 2 - Valores de nitrito residual (em $\mathrm{mg} / \mathrm{kg}$ ) detectados pela técnica de Gries-Ilosvay em amostras de produtos cárneos fabricados sob inspeção federal no Estado do Paraná, de março a julho de 2006

\begin{tabular}{lcccc}
\hline \multicolumn{1}{c}{ Produto* } & $\begin{array}{c}\text { Quantidade de amostras em } \\
\text { não conformidade }\end{array}$ & $\begin{array}{c}\text { Valor } \\
\text { médio }\end{array}$ & $\begin{array}{c}\text { Valor } \\
\text { minimo }\end{array}$ & $\begin{array}{c}\text { Valor } \\
\text { máximo }\end{array}$ \\
\hline Apresuntado (8) & 0 & 54,39 & 18,2 & 90,8 \\
Lanche (3) & 0 & 84,93 & 30,2 & 139 \\
Linguiça tipo calabresa (11) & 2 & 95,85 & 20,7 & 293,1 \\
Linguiças frescais (43) & 20 & 212,3 & 3,7 & 1398,5 \\
Mortadela (33) & 0 & 64,88 & 11,4 & 141,5 \\
Presunto cozido (4) & 0 & 50,17 & 28,1 & 72,1 \\
Salsicha(23) & 1 & 77,51 & 6,8 & 170,4 \\
\hline Total (125) & 23 & 91,43 & 17 & 329,34 \\
\hline
\end{tabular}

* Entre parênteses a quantidade de amostras analisadas por produto.

$\mathrm{Na}$ presente pesquisa, as amostras de linguiças frescais foram as que apresentaram maior variação de teores de nitrito residual, desde quantidades ínfimas de $3,7 \mathrm{mg} / \mathrm{kg}$ até quantidades exageradamente acima do limite legal, como $1.398,5 \mathrm{mg} / \mathrm{kg}$. MELO FILHO et al. (2004) também observaram grande variação entre os teores de nitrito residual em 54 amostras de salsichas comercializadas na região metropolitana de Recife, detectando valores de 27,50 a $180,2 \mathrm{mg} / \mathrm{kg}$, com $18 \%$ do total geral das amostras em não conformidade com a legislação vigente. OLIVEIRA et al. (2005) atribuem as oscilações nos teores de nitrito entre fabricantes de produtos cárneos à composição e à qualidade da matéria-prima e dos ingredientes adicionados e aos procedimentos operacionais em cada unidade de produção, que demonstram deficiência de acompanhamento do processo produtivo. Segundo TURRA \& AYUB (1999), grandes variações na utilização de nitritos demonstram falta de metodologia na fabricação de produtos e consequentes prejuízos à sua qualidade, levantando preocupação quanto aos riscos à saúde pública advindos dessa prática.

Os resultados encontrados para a quantificação de amido em salsicha variaram entre $1,71 \% \mathrm{e}$ $10,28 \%$. Nas amostras de mortadela, esses valores variaram de 3,01 a 16,5 \% (Tabela 3). A utilização de amido é regulamentada pelo MAPA, que determina a utilização de no máximo $2 \%$ e $5 \%$ dessa substância em salsichas e mortadelas, respectivamente (BRASIL, 1997). Apenas duas dentre as marcas de salsichas analisadas (41,7\% das amostras) apresentaram conformidade quanto ao teor de amido preconizado pelo regulamento. Nas salsichas, o valor médio das amostras que não estavam em conformidade foi de $4,26 \%$ de amido. Em mortadelas, foi possível evidenciar oito amostras $(30,76 \%)$ em não conformidade com os dispositivos legais, com $8,15 \%$ de amido, em média.

Diversos autores têm evidenciado abuso na utilização de amido na fabricação de salsichas e mortadelas no Brasil. AUED et al. (1990) detectaram valor médio de $4,98 \%$ de amido em amostras de sal- 
sichas de cinco marcas comerciais, empregando a técnica de Lane-Eynon. SOUZA et al. (1990) detectaram 50\% de amostras de salsichas não conformes (média de 2,64\% de amido) e 11\% de amostras de mortadelas não conformes, com 3,28\% de amido em média. Por outro lado, LABANCA et al. (1999) su- gerem que, em salsichas e mortadelas à base de carne de aves, tais abusos tendem a ocorrer menos, haja vista terem analisado amostras desses produtos e encontrado conformidade com a legislação vigente em $100 \%$ das amostras de mortadela e em $90 \%$ das amostras de salsicha.

Tabela 3 - Valores de amido (em \%) detectados pela técnica de Lane-Eynon em amostras de produtos cárneos fabricados sob inspeção federal no Estado do Paraná, de março a julho de 2006

\begin{tabular}{lccccc}
\hline Produto & Marca & Lote 1 & $\begin{array}{c}\text { Conformidade com a } \\
\text { legislação vigente* }\end{array}$ & Lote 2 & $\begin{array}{c}\text { Conformidade com a } \\
\text { legislação vigente* }\end{array}$ \\
\hline Salsicha & A & 2,43 & Não & 1,71 & Sim \\
& B & 2,88 & Não & 2,06 & Não \\
& C & 2,04 & Não & 1,87 & Sim \\
& D & 4,59 & Não & 2,71 & Não \\
& E & 6,75 & Não & 10,28 & Não \\
\hline Média & F & 6,43 & Não & 2,5 & Não \\
\hline Mortadela & A & 4,19 & Não & 3,52 & Não \\
& G & 4,35 & Sim & 8,25 & Não \\
& H & 4,85 & Sim & 5,3 & Não \\
& I & 3,95 & Sim & 4,61 & Sim \\
& J & 4,29 & Sim & 4,01 & Sim \\
& C & 3,61 & Sim & 3,71 & Sim \\
& D & 7,84 & Sim & 3,21 & Sim \\
& K & 6,62 & Não & 4 & Sim \\
& L & 10,28 & Não & 5,25 & Não \\
& M & 4,55 & Não & 16,5 & Não \\
& E & 3,01 & Sim & 3,34 & Sim \\
& N & 4,85 & Sim & 5,14 & Não \\
& F & 4,24 & Sim & 4,19 & Sim \\
& & 5,13 & Sim & 3,87 & Não \\
\hline
\end{tabular}

* (BRASIL, 1997)

Cabe ressaltar que, sendo detectadas não conformidades microbiológicas e físico-químicas nas amostras dos produtos analisadas, foram lavrados autos de infração contra os respectivos estabelecimentos produtores, por infração aos artigos 372 (excesso de nitrito residual), 414 (excesso de amido) e/ou 422 (presença de micro-organismos patogênicos) do RIISPOA (BRASIL, 1997), sendo ainda os infratores notificados a recolher os lotes dos produtos cujas amostras se apresentaram irregulares. No entanto, o RIISPOA em seu artigo 422 (inciso 9), não se reporta à presença de coliformes fecais, con- siderando como impróprios para consumo os produtos em que se constate a presença de Escherichia coli, que não foi determinada na presente pesquisa. Assim, foram considerados como impróprias as amostras de produtos com coliformes fecais acima do valor máximo permitido (acima de $103 \mathrm{NMP} / \mathrm{g}$ ) pela Agência Nacional de Vigilância Sanitária (BRASIL, 2001).

Porém, a boa qualidade microbiológica encontrada nas amostras de produtos examinadas na presente pesquisa pode não significar a realidade do produto exposto à venda, uma vez que as coletas fo- 
ram realizadas diretamente na indústria, não levando em consideração as possíveis contaminações e/ou multiplicação microbiana durante o transporte e armazenamento nos locais de venda.

\section{CONCLUSÕES}

Há grande heterogeneidade entre as formulações de produtos cárneos das indústrias do Paraná, sendo aconselhável a revisão dos critérios de utilização de nitrito, a fim de preservar a saúde pública. As linguiças frescais produzidas no Estado do Paraná são potencialmente mais perigosas do ponto de vista microbiológico quando comparados com produtos cárneos cozidos.

\section{REFERÊNCIAS}

AUED, S.; CARVALHO, J.B.; TAVARES, M.; ZANELATTO, A.M.; BACETTI, L.B. Determinação de amido em salsichas: comparação entre os métodos de Fehling e de Somogyi-Nelson e avaliação de metodologia para extração do amido. Revista do Instituto Adolfo Lutz, v. 50, n. 1/2, p.251-256, 1990.

BRASIL. AGÊNCIA NACIONAL DE VIGILÂNCIA SANITÁRIA. Resolução RDC 12. Regulamento técnico sobre padrões microbiológicos para alimentos. Diário Oficial da União (seção 1), Brasília, 02/01/2001.

BRASIL. Decreto 2.244/1997. Altera dispositivos do Decreto 30.691/1952, que aprovou o Regulamento da Inspeção Industrial e Sanitária de Produtos de Origem Animal. Diário Oficial da União (seção 1), Brasília, 05/06/1997.

BRASIL. MINISTÉRIO DA AGRICULTURA E DO ABASTECIMENTO. Instrução Normativa nº. 20/1999. Métodos analíticos físico-químicos para controle de produtos cárneos e seus ingredientes, sal e salmoura. Diário Oficial da União (seção 1), Brasília, 09/09/1999.

BRASIL. MINISTÉRIO DA AGRICULTURA, PECUÁRIA E ABASTECIMENTO. Instrução Normativa $n^{\circ}$. 62/2003. Métodos analíticos oficiais para análises microbiológicas para controle de produtos de origem animal e água. Diário Oficial da União (seção 1), Brasília, 18/09/2003.

BRASIL. MINISTÉRIO DA AGRICULTURA, PECUÁRIA E ABASTECIMENTO. Instrução Normativa $\mathrm{n}^{\circ}$. 51/2006. Regulamento técnico de atribuição de aditivos e seus limites das seguintes categorias de alimentos: grupo 8 - carnes e produtos cárneos. Diário oficial da União (seção 1), Brasília, 04/01/2007.
CUNHA, F.A.; CARVALHO, T.M.J.P.; MENEZES, E.A.; OLIVEIRA, M.S.C.; SOUZA, P.A.S.; PEREIRA, A.F.; OLIVEIRA, A.B. Determinação de nitritos em alimentos cárneos. Revista Brasileira de Análises Clínicas, Rio de Janeiro, v.35, n.1, p.3-4, 2003.

DAGUER, H. A cura da carne e a formação de nitrosaminas. Higiene Alimentar, São Paulo, v.19, n.134, p.15-20, 2005.

JAY, J. M. Modern Food Microbiology. Gaithersburg: Aspen Publishers, 2000.

LABANCA, R.A.; SILVA, C.M.G.; GLORIA, M.B.A. Starch levels in refrigerated and frozen chicken based meat products. Brazilian Archives of Biology and Technology, v.42, n,2, p.181-186, 1999.

MARQUES, S.C.; BOARI, C.A.; BRCKO, C.C.; NASCIMENTO, A.R.; PICCOLI, R.H. Avaliação higiênico-sanitária de linguiças tipo frescal comercializadas nos municípios de Três corações e Lavras-MG. Ciência e agrotecnologia, v.30, n.6, p.11201123, 2006.

MELO FILHO, A.B.; BISCONTINI, T.M.B.; ANDRADE, S.A.C. Níveis de nitrito e nitrato em salsichas comercializadas na região metropolitana do Recife. Ciência e Tecnologia de Alimentos, Campinas, v.24, n.3, p.390-392, 2004.

OLIVEIRA, M.J.; ARAÚJO, W.M.; BORGO, L.A. Quantificação de nitrato e nitrito em lingüiças do tipo frescal. Ciência e Tecnologia de Alimentos, Campinas, v.25, n.4, p.736-742, 2005.

PARDI, M.C.; SANTOS, I.F.; SOUZA, E.R.; PARDI, H.S. Ciência, higiene e tecnologia da carne. Goiânia: CEGRAF-UFG / Niterói: EDUFF, 1995. 1110p.

SALVATORI, R.U.; BESSA, M.J.; CARDOSO, M.R.I. Qualidade sanitária de embutidos coletados no mercado público central de Porto Alegre-RS. Ciência Rural, Santa Maria, v.33, n.4, p.771-773, 2003.

SILVA, W.P; GANDRA, E.A.; DUVAL, E. H.; JANTZEN, M. M.; TESSMANN, C.; LIMA, A.S. Qualidade microbiológica de linguiças mistas do tipo frescal produzidas na cidade de Pelotas (RS). Boletim CEPPA, v.20, n.2, p.257-266, 2002.

SOUZA, P.A.; SOUZA, H.B.A.; FALEIROS, R.R.S. Avaliação química de algumas marcas de salsichas e mortadelas. Ciência e Tecnologia de Alimentos, v.10, n.1, p.109-119, 1990.

TURRA, M.; AYUB, M.A.Z. Estudo da variação do teor de nitritos e nitratos em embutidos coloniais: possíveis implicações para a saúde pública. Revista do Instituto Adolfo Lutz, São Paulo, v.58, n.2, p.113-120, 1999. 